\title{
Pseudo-Goldstone Higgs production via gluon fusion
}

\author{
Adam Falkowski ${ }^{1,2}$ \\ ${ }^{1}$ CERN Theory Division, CH-1211 Geneva 23, Switzerland \\ ${ }^{2}$ Institute of Theoretical Physics, Warsaw University, Hoża 69, 00-681 Warsaw, Poland
}

(Received 19 December 2007; published 24 March 2008)

\begin{abstract}
The gluon-gluon-Higgs amplitude is investigated in the context of 5D models of gauge-Higgs unification. A simple algorithm allows one to include, in a fully analytical way, the contribution of the whole Kaluza-Klein tower of a 5D quark to the amplitude. This algorithm is applied to realistic models based on $S O(5)$ symmetry. Within the studied classes of models, the Higgs production cross section is always suppressed.
\end{abstract}

DOI: $10.1103 /$ PhysRevD.77.055018

PACS numbers: $14.80 . \mathrm{Cp}, 11.10 . \mathrm{Kk}$

\section{INTRODUCTION}

The Higgs boson will soon be copiously produced in the LHC, or so we believe. Within the standard model (SM), the dominant production mechanism in hadron colliders is the gluon fusion [1,2]. This process is known to be particularly sensitive to new physics. New colored particles at the $\mathrm{TeV}$ scale or below may significantly alter the SM predictions for the gluon-fusion amplitude. The hierarchy problem of the SM strongly suggests that such new physics states do exist and that they have sizable couplings to the Higgs boson. The examples thoroughly studied in the literature include squarks in supersymmetry, vectorlike quarks in little Higgs, and Kaluza-Klein (KK) quarks in higher dimensional scenarios.

In this paper, I investigate the one-loop gluon-gluonHiggs amplitude in 5D models of the gauge-Higgs unification [3], which provides a dual realization of the pseudogoldstone boson Higgs scenario in 4D [4,5]. Several phenomenological aspects of the gauge-Higgs unification has been studied in the literature [6-11]. One important conclusion of these studies is that the couplings of the Higgs boson to the electroweak gauge bosons and to the SM fermion are modified as a consequence of the origin of the Higgs boson from the fifth component of a gauge field. This observation is relevant for the gluon-fusion amplitude that depends, in particular, on the Yukawa coupling of the Higgs boson to the top quark. Furthermore, the 5D model contains KK partners of the top quark that also couple to the Higgs boson. In 5D gauge-Higgs unification, these couplings are constrained by the fact that the radiatively generated Higgs potential must be free of divergences. I will show that the intricate structure of the Higgs couplings leads to very robust predictions concerning the gluonfusion amplitude.

In Sec. II, I describe a simple algorithm for computing the one-loop contribution of a full KK tower to the gluonfusion amplitude. When all quarks in the tower are sufficiently heavy, $2 m_{n}>m_{\text {Higgs }}$, the result can be expressed in terms of a low-energy limit of the UV brane-to-brane propagators. Thus, the task is reduced to finding mixed 4D momentum/5D position space propagators, which can be formally solved in an arbitrary 5D warped background. The bottom line is that the gluon-fusion amplitude can be calculated analytically, without resorting to numerical methods.

In Sec. III, this algorithm is applied to realistic models based on $S O(5)$ gauge symmetry in 5D [5,12]. The final result for the gluon-fusion amplitude turns out to be surprisingly simple and does not depend on fine-grained details of the model. The only continuous parameter that enters the result is the global symmetry breaking scale $f$. The result depends also on the embedding of the third generation quark sector into multiplets of the $S O(5)$ gauge symmetry. For the two embeddings studied in this paper, the gluon-fusion amplitude is suppressed with respect to the SM prediction. This is a manifestation of the more general conjecture [13] that, in models solving the naturalness problem of the SM, the Higgs production cross section is diminished. The reason is that cancellation of quadratic divergences implies a particular structure of the Yukawa couplings of the Higgs to the quarks, from which the suppression can be deduced [13]. The new element here is that the suppression can be quantified and depends on just one global parameter of the 5D model. In Sec. IV, I show that the same conclusions are reached in the framework of 4D effective theories describing the lightest fermionic KK modes.

Some consequences for Higgs physics at the LHC are pointed out in Sec. V. The Higgs production cross section can be significantly reduced, even down to $30 \%$, for the range of parameters suggested by naturalness and electroweak precision tests.

\section{GLUON FUSION IN 5D GAUGE-HIGGS UNIFICATION}

This section contains a general discussion of the gluongluon-Higgs amplitude in 5D models of gauge-Higgs unification.

The action for a 5D fermion multiplet reads

$$
S_{5}=\int d^{4} x \int_{0}^{L} d y \sqrt{g}\left\{\bar{\Psi}\left(i \Gamma^{N}\left(D_{N}-i g_{5} A_{N}\right)-M\right) \Psi\right\} .
$$


Here $M$ is the bulk mass. $\Psi$ is a quark multiplet-a triplet under color $S U(3)$. It is also charged under another group factor that contains the SM electroweak group so that it may contain several quark flavors with top, bottom, or exotic quantum numbers. We will study this action in a general warped background with the line element $d s^{2}=$ $a^{2}(y) d x^{2}-d y^{2}$.

The fifth component of the gauge field may host physical degrees of freedom that, in the KK picture, become massless (at tree level) 4D scalar fields. If the 5D gauge group is nonabelian, a vev of these scalars leads to spontaneous breaking of gauge symmetry. We single out one generator $T^{\hat{a}}$ along which the vev resides and we define the Higgs boson field $h(x)$ as oscillations around the vev,

$$
A_{5} \rightarrow \frac{a^{-2}(y)}{\left[\int_{0}^{L} a^{-2}\right]^{1 / 2}} T^{\hat{a}}(\tilde{v}+h(x)) .
$$

The normalization factors are chosen such as to make the Higgs $h(x)$ canonically normalized in the KK picture and $\tilde{v}$ is the Higgs vev. In 5D gauge-Higgs unification, electroweak breaking is characterized by the Higgs vev and by another scale $f$ called the global symmetry breaking scale. The latter scale depends on the parameters of the 5D model,

$$
f=\frac{\sqrt{2}}{g_{5}\left(\int_{0}^{L} a^{-2}\right)^{1 / 2}} .
$$

The $W$ boson mass depends on both $\tilde{v}$ and $f$,

$$
m_{W}=\frac{g_{L} f \sin (\tilde{v} / f)}{2},
$$

so that $v=f \sin (\tilde{v} / f)=246 \mathrm{GeV}$ is the electroweak breaking scale. The ratio $v / f$ will play an important role in the following: the suppression of the gluon-fusion amplitude will depend on it.

We expand the fermions into the KK mass eigenstates

$$
\begin{aligned}
& \Psi_{L}(x, y)=f_{L, n}(\tilde{v}, y) \Psi_{L, n}(x) \\
& \Psi_{R}(x, y)=f_{R, n}(\tilde{v}, y) \Psi_{R, n}(x) .
\end{aligned}
$$

The profiles satisfy the equations of motion,

$$
\begin{aligned}
\left(\hat{D}_{5}+M\right) f_{R, n}(\tilde{v}, y) & =m_{n} a^{-1} f_{L, n}(\tilde{\boldsymbol{v}}, y) \\
\left(-\hat{D}_{5}+M\right) f_{L, n}(\tilde{v}, y) & =m_{n} a^{-1} f_{R, n}(\tilde{v}, y),
\end{aligned}
$$

and boundary conditions appropriate for the model. Furthermore, they satisfy the orthonormality conditions from which follow the completeness relation

$$
\begin{aligned}
\sum_{n} f_{L, n}(\tilde{\boldsymbol{v}}, z) f_{L, n}^{\dagger}(\tilde{\boldsymbol{v}}, y) & =\sum_{n} f_{R, n}(\tilde{\boldsymbol{v}}, z) f_{R, n}^{\dagger}(\tilde{\boldsymbol{v}}, y) \\
& =a^{-3}(y) \delta(y-z) I,
\end{aligned}
$$

where $I$ is the identity matrix in the flavor space.

The Higgs boson couples to the fermionic eigenstates as $-y_{n m} h \bar{\psi} \psi_{R, m}$, where the Yukawa couplings are given by

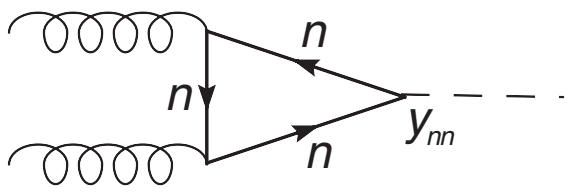

FIG. 1. Contribution to the gluon-gluon-Higgs amplitude of the $n$th fermionic KK eigenstate. Because the QCD coupling of quark eigenstates to gluons is diagonal and the eigenstates do not mix with each other, only diagonal Yukawa couplings are relevant.

$$
y_{n m}=-i g_{5} \frac{\int_{0}^{L} a^{2}(y) f_{L, n}^{\dagger}(\tilde{v}, y) T^{\hat{a}} f_{R, n}(\tilde{v}, y)}{\left(\int_{0}^{L} a^{-2}(y)\right)^{1 / 2}} .
$$

Although it is not obvious at this point, the couplings $y_{n m}$ are real.

The objective is to compute the one-loop contribution of the fermionic eigenstates to the gluon-gluon-Higgs amplitude. The relevant Feynman diagram is depicted on Fig. 1. In the SM, this amplitude is dominated by the top quark who has the largest Yukawa coupling to the Higgs. Assuming the Higgs boson is light enough, $m_{\text {higgs }}<2 m_{t}$, the amplitude can be written as

$$
\mathcal{M}_{\mathrm{ggh}}(\mathrm{SM}) \approx f(\epsilon, p) \frac{y_{t t}}{m_{t}}=f(\epsilon, p) \frac{1}{v},
$$

where $f(\epsilon, p)$ depends on the momenta and polarizations of the incoming gluons and its precise form will be of no importance. In the following we always assume that $m_{\text {Higgs }}<2 m_{n}$, for the top quark as well as for all new physics quarks. This is a safe assumption in the $5 \mathrm{D}$ pseudogoldstone scenario: the Higgs potential is generated at one loop so that the Higgs boson is expected to be light, not far from the present direct search limit. Thus, the amplitude can be approximated by

$$
\mathcal{M}_{\mathrm{ggh}}(5 \mathrm{D}) \approx f(\epsilon, p) \sum_{n} \frac{y_{n n}}{m_{n}},
$$

where the sum goes over all heavy enough fermionic eigenstates (it includes the SM top quark, but not the bottom or any of the lighter quarks).

Now we prove a remarkable sum rule. We first note that, using the equations of motion, the profiles can be represented as

$$
\begin{aligned}
f_{R, n}(\tilde{\boldsymbol{v}}, y)= & \Omega(y) a^{-2}(y) e^{-M y}\left(f_{R, n}(\tilde{\boldsymbol{v}}, 0)\right. \\
& \left.+m_{n} \int_{0}^{y} a\left(y^{\prime}\right) e^{M y^{\prime}} \Omega^{-1}\left(y^{\prime}\right) f_{L, n}\left(\tilde{\boldsymbol{v}}, y^{\prime}\right)\right) \\
f_{L, n}(\tilde{\boldsymbol{v}}, y)= & \Omega(y) a^{-2}(y) e^{M y}\left(f_{L, n}(\tilde{\boldsymbol{v}}, 0)\right. \\
& \left.-m_{n} \int_{0}^{y} a\left(y^{\prime}\right) e^{-M y^{\prime}} \Omega^{-1}\left(y^{\prime}\right) f_{R, n}\left(\tilde{\boldsymbol{v}}, y^{\prime}\right)\right),
\end{aligned}
$$


where $\Omega(y)=e^{i g_{5}} \int_{0}^{y}\left\langle A_{5}\right\rangle$. Using the above expressions and the completeness relations one can derive

$$
\sum_{n} \frac{y_{n n}}{m_{n}}=-i g_{5}\left[\int_{0}^{L} a^{-2}\right]^{1 / 2} \sum_{n} m_{n}^{-1} f_{L, n}^{\dagger}(\tilde{v}, 0) T^{\hat{a}} f_{R, n}(\tilde{v}, 0) .
$$

The sum in the last expression is related to the chiralityflipping propagator in $4 \mathrm{D}$ momentum/5D position space. More precisely, the propagator is defined by

$$
P_{R L}(y, z)=i \sum_{n} f_{R, n}(y) f_{L, n}^{\dagger}(z) \frac{m_{n}}{p^{2}-m_{n}^{2}},
$$

so that we can rewrite

$$
\sum_{n} \frac{y_{n n}}{m_{n}}=\left.f^{-1} \operatorname{Tr}\left[\sqrt{2} T^{\hat{a}} P_{R L}(0,0)\right]\right|_{p^{2} \rightarrow 0},
$$

where the scale $f$ was defined in Eq. (2.3). In the SM, the amplitude in the decoupling limit is proportional to $1 / m_{t}$, which is the zero momentum limit of the top quark propagator. In $5 \mathrm{D}$, this is generalized to the zero momentum limit of the UV boundary propagator. This kind of result could be expected from holography. In the end, the 5D setup can be interpreted as a dual description of 4D fundamental quarks (living on the UV boundary) that mix with fermionic composite operators from a strongly coupled sector [14]. It should be underlined, however, that Eq. (2.14) is not a "holographic prescription," but a rigorous result derived from the 5D formalism.

Finally, we define $R_{g}^{1 / 2}$ as the ratio of the gluon-gluonHiggs amplitude in the 5D model to that in the SM (with $\left.m_{h}<2 m_{t}\right)$. The ratio is given by the following simple expression

$$
R_{g}^{1 / 2}=\frac{v}{f}\left(\left.\operatorname{Tr}\left[\sqrt{2} T^{\hat{a}} P_{R L}(0,0)\right]\right|_{p^{2} \rightarrow 0}-\sum_{\text {light }} \frac{y_{n n}}{m_{n}}\right) .
$$

The methods of computing fermionic propagators in a warped background are reviewed in Appendix A. In the next section, we apply Eq. (2.15) in the context of realistic 5D models of pseudogoldstone Higgs.

\section{III. $S O(5)$ MODELS}

We apply the general methods outlined in the previous section in the context of 5D models with the electroweak group embedded in $S O(5) \times U(1)_{X}$ [5]. This is the simplest setup that accommodates the correct Weinberg angle and the custodial symmetry. The latter is indispensable in 5D warped models in order to keep the Peskin-Takeuchi $T$ parameter under control $[8,15]$. $S O(5)$ has 10 generators: three form the $S U(2)_{L}$ subgroup (identified with the standard model $S U(2)$ ), another three form the $S U(2)_{R}$ subgroup (identified with the custodial symmetry), and the remaining four generators $T_{C}^{b}$ belong to the $S O(5) / S O(4)$ coset. Some useful facts about $S O(5)$ are collected in Appendix B.

The $S O(5)$ gauge symmetry is reduced on the boundaries by imposing Dirichlet boundary conditions for some of the generators. The surviving gauge symmetry on the UV brane is $S U(2)_{L} \times U(1)_{Y}$, the hypercharge being a linear combination of the $T_{R}^{3}$ and $U(1)_{X}$ generators. On the IR brane, the symmetry is reduced down to $S O(4)=$ $S U(2)_{L} \times S U(2)_{R}$. The four generators from the $S O(5) / S O(4)$ coset have Dirichlet boundary conditions on both branes, so that the fifth components of the corresponding gauge fields hosts scalar fields identified with the SM Higgs doublet. The vev is chosen along the $T_{C}^{4}$ generator. The electroweak breaking scale is $v=f \sin (\tilde{v} / f)$, where $f$ is defined in Eq. (2.3).

There are several options for embedding the third generation quarks into $S O(5)$ multiplets. The first model we consider here is a variation on that introduced in Ref. [5]. The top and bottom quarks are embedded into two 5D quarks in the spinorial representation $\mathbf{4}_{\mathbf{1} / \mathbf{6}}$,

$$
\begin{aligned}
& Q_{1}=\left(q_{1}, q_{1}^{c}\right)=\left(t_{1}, b_{1}, t_{1}^{c}, b_{1}^{c}\right) \\
& Q_{2}=\left(q_{2}, q_{2}^{c}\right)=\left(t_{2}, b_{2}, t_{2}^{c}, b_{2}^{c}\right) .
\end{aligned}
$$

The IR boundary conditions are the same for $Q_{1}$ and $Q_{2}$,

$$
q_{R, i}(L)=q_{L, i}^{c}(L)=0 .
$$

The UV boundary conditions are chosen as ${ }^{1}$

$$
\begin{array}{cc}
\theta_{2} q_{1, R}(0)-\theta_{1} q_{2, R}(0)=0 & \bar{\theta}_{1} q_{1, L}(0)+\bar{\theta}_{2} q_{2, L}(0)=0 \\
t_{1, L}^{c}(0)=t_{2, R}^{c}(0)=0 & b_{1, R}^{c}(0)=b_{2, L}^{c}(0)=0 .
\end{array}
$$

The KK towers include two quark eigenstates that become massless in the limit of no electroweak breaking. These are identified with the SM top and bottom quarks. The mass splitting between the top and the bottom quark can achieved if $\left|\theta_{1} / \theta_{2}\right| \ll 1$ or if $M_{1}>M_{2}$, in which case $m_{b}^{2} / m_{t}^{2} \approx\left|\theta_{1} / \theta_{2}\right|^{2} \int_{0}^{L} a^{-1} e^{-2 M_{1} y} / \int_{0}^{L} a^{-1} e^{-2 M_{2} y}$.

In this scenario, all the quark eigenstates couple to the Higgs boson and contribute to the gluon-gluon-Higgs amplitude. We first compute the contribution of the top quark tower. Using Eq. (2.14),

$$
\sum_{\text {top }} \frac{y_{n n}}{m_{n}}=\left.\frac{1}{2 f}\left(P_{R L}^{t_{1} t_{1}^{c}}(0,0)+P_{R L}^{t_{1}^{c} t_{1}}(0,0)+P_{R L}^{t_{2} t_{2}^{c}}(0,0)\right)\right|_{p^{2}=0} .
$$

We compute the UV propagators using the algorithm outlined in Appendix A. The final result is very simple,

\footnotetext{
${ }^{1}$ The peculiar UV boundary conditions for the electroweak doublets $q_{i}$ can be realized by mixing the linear combination $\bar{\theta}_{1} q_{1, L}+\bar{\theta}_{2} q_{2, L}$ with a UV boundary fermion $\tilde{q}_{R}$ through a boundary mass term, and taking the boundary mass to infinity.
} 


$$
\sum_{\text {top }} \frac{y_{n n}}{m_{n}}=\frac{\cos (\tilde{v} / f)}{f \sin (\tilde{v} / f)} .
$$

The same result is obtained for $\sum$ bottom $\frac{y_{n n}}{m_{n}}$. In that case, however, the sum is almost entirely dominated by the lightest bottom quark contribution. More precisely, starting from Eq. (2.8) one finds $y_{b b} / m_{b}=f^{-1} \cot (\tilde{v} / f)+\mathcal{O}\left(m_{b}^{2}\right)$. Thus, by Eq. (2.15), the bottom quark tower does not contribute significantly to the gluon-gluon-Higgs amplitude. We conclude that in this model based on the spinorial representation

$$
R_{g}^{1 / 2} \approx \cos (\tilde{v} / f)+\mathcal{O}\left(m_{b}^{2} / M_{\mathrm{KK}}^{2}\right) \approx \sqrt{1-v^{2} / f^{2}} .
$$

In the second model, we consider the third generation is embedded in the fundamental $S O(5)$ representation [12]. We consider two 5D quarks $Q_{1}, Q_{2}$ transforming as $\mathbf{5}_{\mathbf{2} / \mathbf{3}}$, $5_{-1 / 3}$, respectively. The quantum numbers are embedded into the fiveplet as

$$
Q_{1}=\frac{1}{\sqrt{2}}\left(\begin{array}{c}
\chi+b_{1} \\
i\left(\chi-b_{1}\right), \\
t_{1}+\tilde{t} \\
i\left(t_{1}-\tilde{t}\right) \\
\sqrt{2} t^{c}
\end{array}\right) \quad Q_{2}=\frac{1}{\sqrt{2}}\left(\begin{array}{c}
\rho+t_{2} \\
i\left(\rho-t_{2}\right) \\
b_{2}+\tilde{b} \\
i\left(b_{2}-\tilde{b}\right) \\
\sqrt{2} b^{c}
\end{array}\right) .
$$

The exotic quarks $\chi$ and $\rho$ have electric charges $5 / 3$ and $-4 / 3$, respectively. The upper four components in each multiplet can be collected into bidoublets of $S U(2)_{L} \times$ $S U(2)_{R}: \Phi_{i}=\left(q_{i}, \tilde{q}_{i}\right)$, where $q_{i}=\left(t_{i}, b_{i}\right), \quad \tilde{q}_{1}=(\chi, \tilde{t})$, $\tilde{q}_{2}=(\tilde{b}, \rho)$. The fact that the top-bottom $S U(2)_{L}$ doublet is embedded into a bidoublet protects the SM $Z \bar{b} b$ vertex against dangerous corrections [16], which provides rationale for this more complicated model.

The IR boundary conditions are chosen as

$$
\Phi_{R, 1}(L)=\Phi_{R, 2}(L)=0 \quad t_{L}^{c}(L)=b_{L}^{c}(L)=0 .
$$

The UV boundary conditions are

$$
\begin{array}{r}
\theta_{2} q_{1, R}(0)-\theta_{1} q_{2, R}(0)=0 \\
\bar{\theta}_{1} q_{1, L}(0)+\bar{\theta}_{2} q_{2, L}(0)=0 \\
t_{L}^{c}(0)=b_{L}^{c}(0)=0 \\
\tilde{q}_{1, L}(0)=\tilde{q}_{2, L}(0)=0 .
\end{array}
$$

The exotic quarks do not couple to the Higgs boson at all. Furthermore, using the same arguments as previously, one can prove that the bottom quark tower contribution to gluon-gluon-Higgs amplitude is suppressed by the small bottom quark mass. As for the top quark tower contribution, we compute

$$
\sum_{t o p} \frac{y_{n n}}{m_{n}}=\left.\frac{1}{\sqrt{2} f}\left(-P_{R L}^{t^{c} t_{1}}+P_{R L}^{t^{c} \tilde{t}}\right)\right|_{p^{2}=0}=\frac{\cos (\tilde{v} / f)}{f \sin (\tilde{v} / f)} .
$$

This is the same result as that in obtained in the spinorial model. However, the present setup is incomplete as it stands, because it does not have a correct electroweak breaking vacuum. In the language of Ref. [17], the spectral function corresponding to the top quark tower is $\rho \approx$ $S_{M_{1}}(L) S_{-M_{1}}(L)-(1 / 2) \sin ^{2}(\tilde{v} / f)$ and the minimum of the Higgs potential falls at $\sin ^{2}(\tilde{v} / f)=1$, which implies that the model is equivalent, in practice, to a Higgsless theory $[9,18]$. In order to achieve $\sin ^{2}(\tilde{v} / f) \ll 1$, as suggested by electroweak precision tests, we need to introduce the so-called shadow multiplet, whose role is to produce a quartic term, $\sin ^{4}(\tilde{v} / f)$, in the spectral function $[5,19,20]$. A shadow multiplet is another 5D quark that has no light modes in the limit of no electroweak breaking; massless modes appear however for maximal electroweak breaking. Here we consider a $5 \mathrm{D}$ quark $S$ transforming as $\mathbf{5}_{\mathbf{2} / \mathbf{3}}$. It contains a bidoublet $\Phi^{s}$ and a singlet $t^{s}$ with the boundary conditions chosen as

$$
\Phi_{R}^{s}(0)=t_{L}^{s}(0)=\Phi_{L}^{s}(L)=t_{R}^{s}(L)=0 .
$$

In this setup, the contribution of the new shadow top quark tower to the gluon-gluon-Higgs amplitude is given by

$$
\sum_{\text {shadow }} \frac{y_{n n}}{m_{n}}=-\frac{\sin (\tilde{v} / f)}{f \cos (\tilde{v} / f)} .
$$

The minus sign implies that the shadow tower interferes destructively with that of the top. At the end of the day, we find that in this model based on the fundamental representation

$$
R_{g}^{1 / 2} \approx \frac{\cos (2 \tilde{v} / f)}{\cos (\tilde{v} / f)} \approx \frac{1-2 v^{2} / f^{2}}{\sqrt{1-v^{2} / f^{2}}} .
$$

The shadow multiplet can mix with $Q_{1}$ and $Q_{2}$ via IR boundary mass terms. Including these mass terms does not change the above result. If another shadow multiplet is included, for example $\mathbf{5}_{-\mathbf{1 / 3}}$ as in [12], it would further diminish the amplitude.

One could repeat the same procedure for other gaugeHiggs unification models, e.g., for $S O(5)$ models with the third generation embedded in the adjoint representation or for models based on the $S U(3)$ gauge group. The recurring feature of the gluon-fusion amplitude is that the ratio $R_{g}$ depends only on $v / f$ and is not sensitive to the details of the KK quark spectrum. Furthermore, in all cases one finds $R_{g}^{1 / 2}<1$.

\section{4D EFFECTIVE DESCRIPTION}

The results for the gluon-gluon-Higgs amplitude that were obtained in $5 \mathrm{D}$, can be reproduced in a $4 \mathrm{D}$ framework. In the spirit of Refs. [18,21,22], one can construct a 4D model that mimics the low-energy dynamics of 5D gauge-Higgs models. The gauge group is the SM one, $S U(3)_{C} \times S U(2)_{L} \times U(1)_{Y}$, while the bulk gauge group of the $5 \mathrm{D}$ setup is realized as an approximate global 
symmetry, which is spontaneously broken by a vev of a scalar field. The pseudogoldstone Higgs resides in that scalar field. The quark sector includes the standard model quarks and a finite number of vectorlike quarks. The 4D setup can be considered as an effective low-energy description of the 5D gauge-Higgs unification setup (or some 4D strong dynamics), with the cutoff identified with the resonance scale.

In 4D, Yukawa couplings of the Higgs boson to the quarks are given by $y_{n m}=\frac{\partial M_{n m}}{\partial \tilde{v}}$, where $M$ is the quark mass matrix and $\tilde{v}$ is the Higgs vev. Since we deal with a finite number of quarks, it is more handy to adopt another sum rule to compute the quark contributions to the gluongluon-Higgs amplitude. We can rewrite

$$
\begin{aligned}
\sum \frac{y_{n n}}{m_{n}} & =\operatorname{Tr}\left(y M^{-1}\right)=\operatorname{Tr}\left(\frac{\partial M}{\partial \tilde{v}} M^{-1}\right)=\frac{\partial \operatorname{Tr} \log M}{\partial \tilde{v}} \\
& =\frac{\partial \log \operatorname{det} M}{\partial \tilde{v}} .
\end{aligned}
$$

Thus, the gluon-gluon-Higgs amplitude can be related to the determinant of the quark mass matrix [23],

$$
R_{g}^{1 / 2}=v \frac{\partial}{\partial \tilde{v}} \log \operatorname{det} M(\tilde{v})
$$

In fact, this formula can be also applied to 5D gauge-Higgs models, in spite of the fact that $\operatorname{det} M(\tilde{v})$ diverges in 5D due to infinite multiplicity of KK states. ${ }^{2}$ In the gauge-Higgs case, we should replace $\operatorname{det} M(\tilde{v})$ in Eq. (4.2) with $\rho^{1 / 2}(0)$, where $\rho\left(p^{2}\right) \equiv \operatorname{det}\left(-p^{2}+m_{n}^{2}\right)$ is the spectral function that can be computed by solving the $5 \mathrm{D}$ equations of motion $[17,20]$. This method yields the same results as the position propagator method used in Sec. II.

The 4D effective description of the 5D model based on the $S O(5)$ spinorial representation is the following [18] (see also [21]). Left-chirality quarks include two $S U(2)_{L}$ doublets $q_{L, i}=\left(t_{L, 1}, b_{L, i}\right)$, one singlet top $T_{L}^{c}$, and one singlet bottom $B_{L}^{c}$. Right-chirality quarks include two top singlets $t_{R, i}^{c}$, two bottom singlets $b_{R, i}^{c}$, and one doublet $Q_{R}=\left(T_{R}, B_{R}\right)$. This amounts to three top Dirac states, another three bottom Dirac states and no exotics. The quarks are collected into $S O(5)$ spinors as follows:

$$
\Psi_{L}=\left(\begin{array}{c}
q_{L, 1} \\
-i B_{L}^{c} \\
-i T_{L}^{c}
\end{array}\right) \quad \Psi_{R}=\left(\begin{array}{c}
Q_{R} \\
i b_{R, 1}^{c} \\
i t_{R, 1}^{c}
\end{array}\right) .
$$

The global $S O(5)$ symmetry is spontaneously broken by a scalar field transforming as $\mathbf{5}_{\mathbf{0}}$. The pseudogoldstone Higgs is embedded in that scalar as $\Phi_{M} \rightarrow$ $f(0,0,0, \sin (\tilde{v} / f), \cos (\tilde{v} / f))$. The Yukawa couplings re-

\footnotetext{
${ }^{2}$ This point was clarified in private discussions with Csabi Csaki and Andy Weiler.
}

spect $S O(5)$. The global symmetry is explicitly yet softly broken by vectorlike mass terms,

$$
\begin{aligned}
-\mathcal{L}_{y u k}= & y \bar{\Psi}_{L} \Gamma^{M} \Phi_{M} \Psi_{R}+f \lambda_{q} \bar{q}_{L, 2} Q_{R}+f \lambda_{t} \bar{T}_{L}^{c} t_{R, 2}^{c} \\
& +f \lambda_{b} \bar{B}_{L}^{c} b_{R, 2}^{c}+\text { H.c. }
\end{aligned}
$$

The resulting mass terms for the top quark can be represented in the matrix,

$$
\begin{gathered}
-\mathcal{L}_{\text {mass }}=\left(\bar{t}_{L, 1}, \bar{t}_{L, 2}, \bar{T}_{L}^{c}\right) M\left(\begin{array}{c}
t_{R, 1}^{c} \\
t_{R, 2}^{c} \\
T_{R}
\end{array}\right) \\
M=f\left(\begin{array}{ccc}
y \sin (\tilde{v} / f) & 0 & y \cos (\tilde{v} / f) \\
0 & 0 & \lambda_{q} \\
y \cos (\tilde{v} / f) & \lambda_{t} & -y \sin (\tilde{v} / f)
\end{array}\right) .
\end{gathered}
$$

The trace of the mass matrix squared does not depend on $\tilde{v}$, which implies that the top quarks generate no quadratically divergent corrections to the Higgs mass parameters at one loop. In fact, this model is supersoft; the logarithmic divergences cancel as well. The determinant of the mass matrix is proportional to $\lambda_{q} \lambda_{t} y \sin (\tilde{v} / f)$. From Eq. (4.2) we find

$$
R_{g}^{1 / 2}=\cos (\tilde{v} / f)
$$

in accord with Eq. (3.6).

Another model provides an effective description to the $5 \mathrm{D}$ model based on the $S O(5)$ fundamental representation [18]. Left-chirality quarks include two $S U(2)_{L}$ doublets $q_{L, i}=\left(t_{L, i}, b_{L, i}\right)$, one singlet $T_{L}^{c}$, and one exotic (hypercharge $7 / 6)$ doublet $\tilde{Q}_{L}=\left(\chi_{L}, \tilde{T}_{L}\right)$. Right-chirality quarks include two singlets $t_{R, i}^{c}$, one doublet $Q_{R}=\left(T_{R}, B_{R}\right)$, and one exotic doublet $\tilde{Q}_{R}=\left(\chi_{R}, \tilde{T}_{R}\right)$. This makes four Dirac quarks with top quantum numbers, one massless chiral bottom quark, one Dirac bottom quark, and one exotic quark with charge $5 / 3$. More structure is needed to give mass to the bottom quark, but we do not elaborate on it here. The quark fields are collected into fives as follows:

$$
\begin{gathered}
\Psi_{L}=\left(\begin{array}{c}
\frac{1}{\sqrt{2}}\left(b_{L}+\chi_{L}\right) \\
-\frac{i}{\sqrt{2}}\left(b_{L}-\chi_{L}\right) \\
\frac{i}{\sqrt{2}}\left(t_{L, 1}-\tilde{T}_{L}\right) \\
\frac{1}{\sqrt{2}}\left(t_{L, 1}+\tilde{T}_{L}\right) \\
T_{L}^{c}
\end{array}\right) \\
\Psi_{R}=\left(\begin{array}{c}
\frac{1}{\sqrt{2}}\left(B_{R}+\chi_{R}\right) \\
-\frac{i}{\sqrt{2}}\left(B_{R}-\chi_{R}\right) \\
\frac{i}{\sqrt{2}}\left(T_{R}-\tilde{T}_{R}\right) \\
\frac{1}{\sqrt{2}}\left(T_{R}+\tilde{T}_{R}\right) \\
t_{R, 2}^{c}
\end{array}\right) .
\end{gathered}
$$

We write down $S O(5)$ symmetric Yukawa couplings and soft-breaking vectorlike mass terms, 


$$
-\mathcal{L}_{y u k}=y_{1} \bar{\Psi}_{L} \Phi t_{R, 1}^{c}+y_{2} \bar{T}_{L}^{c} \Phi^{\dagger} \Psi_{R} f \lambda_{1} \bar{q}_{L, 1} Q_{R}+f \lambda_{2} \bar{q}_{L, 2} Q_{R}+f \lambda_{\chi} \overline{\tilde{Q}}_{L} \tilde{Q}_{R}+\text { H.c. }
$$

Some mass terms allowed by gauge symmetries, e.g., $T_{L}^{c} t_{R, 2}^{c}$, are omitted because they would violate the softness of the global symmetry breaking. The mass matrix for the top quarks,

$$
\begin{gathered}
-\mathcal{L}_{\text {mass }}=\left(\bar{t}_{L, 1}, \bar{t}_{L, 2}, T_{L}^{c}, \overline{\tilde{T}}_{L}\right) M\left(\begin{array}{c}
t_{R, 1}^{c} \\
t_{R, 2}^{c} \\
T_{R} \\
\tilde{T}_{R}
\end{array}\right) \\
M=f\left(\begin{array}{cccc}
y_{1} \sin (\tilde{v} / f) & 0 & \lambda_{1} & 0 \\
0 & 0 & \lambda_{2} & 0 \\
\sqrt{2} y_{1} \cos (\tilde{v} / f) & \sqrt{2} y_{2} \cos (\tilde{v} / f) & y_{2} \sin (\tilde{v} / f) & y_{2} \sin (\tilde{v} / f) \\
y_{1} \sin (\tilde{v} / f) & 0 & 0 & \lambda_{\chi}
\end{array}\right) .
\end{gathered}
$$

The determinant is now proportional to $\sin (2 \tilde{v} / f)$, rather than to $\sin (\tilde{v} / f)$ as in the spinorial realization. In consequence,

$$
R_{g}^{1 / 2}=\frac{\cos (2 \tilde{v} / f)}{\cos (\tilde{v} / f)}
$$

as in Eq. (3.13).

\section{DISCUSSION}

I presented an analysis of the gluon-gluon-Higgs amplitude in two models with a pseudogoldstone Higgs boson realized in $5 \mathrm{D} S O(5)$ gauge-Higgs unification. In both cases, the Higgs production cross section is suppressed with respect to the SM result. Suppression effects were also concluded in a closely related framework of little Higgs [24] (in gauge-Higgs, the suppression was alluded to in Ref. [8]). I did not prove that pseudogoldstone Higgs scenarios with an enhanced Higgs production cross section do not exist. In fact, one can write down a somewhat stretched counterexample where $R_{g}^{1 / 2}<-1$ due to contributions of several shadow multiplets, so that the cross section would be enhanced. The fact is, however, that the simplest setups consistent with naturalness and electroweak precision tests always predict suppression of the Higgs production rate. In contrast, enhancement can be achieved in the parameter space of 5D models in which the Higgs potential is not protected, for example, in universal extra dimensions [25], or in the warped models based on the $S U(2)_{L} \times S U(2)_{R}$ group [26]. Enhancement could also be achieved in the minimal supersymmetric standard model although, in that case, in the most interesting parameter space region with the minimal electroweak finetuning one also finds a suppression [27].

The most interesting result obtained in this paper is that, if $m_{h}<2 m_{n}$ for the top quark and all new physics quarks, the suppression factor $R_{g}$ in 5D models depends very little on the details of the spectrum. One could expect that the result depends on the individual masses of the vectorlike quarks, since the Yukawa coupling of the top quark can be substantially modified in the presence of fairly light new physics states. However, summing up the contributions of the whole KK tower leaves only the dependence on $v / f$ the ratio of the electroweak breaking scale to the global symmetry breaking scale. Thus, the prediction for the gluon-fusion amplitude is a very robust feature of the gauge-Higgs models.

The suppression factor $R_{g}$ depends also on the embedding of the third generation into $S O(5)$ multiplets, of which several options exist in the literature [5,12]. Given that we identify the embedding by observing some of the fermionic resonances, a precise enough measurement of the Higgs production cross section could provide a simple way to determine the scale $f$. The latter is very important phenomenologically, as it controls the growth of the longitudinal gauge boson scattering amplitude below the resonance scale [9]; knowing the scale $f$ would provide an answer to the question if strong WW scattering occurs at the energies accessible at the LHC.

There are two theoretical arguments concerning the actual value of $f$, that hint towards a different range. On one hand, the little hierarchy problem suggests $f$ should not be too large because the fine-tuning needed to achieve $v / f \ll 1$ is proportional to $v^{2} / f^{2}$ [5]. On the other hand, electroweak precision tests suggest a larger value, as $v / f$ of order unity corresponds to an effectively heavy Higgs [18], which is disfavored by electroweak data. Furthermore, we expect $f \geq 500 \mathrm{GeV}$, since a smaller value implies the existence of vector resonances with masses below $3 \mathrm{TeV}$ (to unitarize the WW scattering), which is disfavored by electroweak precision data. With the above facts in mind, I pick up two benchmark points $f=500 \mathrm{GeV}, f=1 \mathrm{TeV}$ corresponding to the finetuning of order $25 \%$ and $5 \%$, respectively, and to the effective Higgs mass 250 and $145 \mathrm{GeV}$ (for the true Higgs mass $115 \mathrm{GeV}$ ). The suppression factor in the two models we studied is given by 


\begin{tabular}{llc} 
& 4 & 5 \\
\hline$f=500 \mathrm{GeV}$ & $R_{g}=75 \%$ & $R_{g}=35 \%$ \\
$f=1000 \mathrm{GeV}$ & $R_{g}=95 \%$ & $R_{g}=82 \%$
\end{tabular}

For a reasonable choice of parameters, the suppression can be particularly large in the model based on the fundamental (5) representation, the one that is favored by the measurements of the $Z \bar{b} b$ vertex. Using the diphoton channel at the LHC, the theoretical estimate of the Higgs production cross section may be confronted with experiment with $\sim 10 \%$ accuracy [2]. Thus, the suppression effect due to the pseudogoldstone nature of the Higgs boson should be confirmed at the LHC in most of the interesting parameter space.

Note that the photon-photon-Higgs amplitude is suppressed too; that amplitude is dominated by a loop of $W$ boson whose coupling to the Higgs boson is suppressed by $\cos (\tilde{v} / f)$ [9]. However, other decay amplitudes, for example $h \rightarrow \bar{b} b$ are typically suppressed too. The modification of the branching ratios depends on the embedding of the SM fields into multiplets of the bulk gauge group. In the two models studies in Sec. III the branching ratios are not changed.

If gauge-Higgs unification is realized in nature, Higgs boson searches at the LHC may be more challenging than previously assumed. The suppression of the Higgs production cross section predicted by theses models may obstruct a quick discovery of the Higgs boson. On the positive side, if fairly light vectorlike quarks are present, decays of these quarks may enhance the Higgs production [8]. Finally, not discovering the Higgs boson after $30 \mathrm{fb}^{-1}$ of data should not be considered discouraging but rather a hint that the pseudogoldstone mechanism is at work.

\section{ACKNOWLEDGMENTS}

I am grateful to Babis Anastasiou for inspiring this work and to Roberto Contino for clarifications on $S O(5)$ models. Thanks to Babis Anastasiou, Ian Low, and Jose Santiago for comments on the manuscript and for pointing out blunders in an earlier version. I also profited from postv1 discussions with Csaba Csaki, Ami Katz, Maria Krawczyk, Eduardo Pontón, Carlos Wagner, Andy Weiler, and Neal Weiner. I am partially supported by the European Community Contract No. MRTN-CT-2004503369 for the years 2004-2008 and by the MEiN Grant No. 1 P03B 09929 for the years 2005-2007.

\section{APPENDIX A: FERMIONIC PROPAGATOR IN GAUGE-HIGGS BACKGROUND}

The propagator can be equivalently defined either as an inverse of the kinetic operator in the Lagrangian or, as in the following, as a sum of KK propagators weighted by profiles. We define the mixed (4D momentum/5D position space) fermionic propagators by

$$
\begin{aligned}
& P_{L L}(y, z)=i \sum_{n} \frac{f_{L, n}(y) f_{L, n}^{\dagger}(z)}{p^{2}-m_{n}^{2}} \\
& P_{R R}(y, z)=i \sum_{n} \frac{f_{R, n}(y) f_{R, n}^{\dagger}(z)}{p^{2}-m_{n}^{2}} \\
& P_{L R}(y, z)=i \sum_{n} \frac{m_{n} f_{L, n}(y) f_{R, n}^{\dagger}(z)}{p^{2}-m_{n}^{2}} \\
& P_{R L}(y, z)=i \sum_{n} \frac{m_{n} f_{R, n}(y) f_{L, n}^{\dagger}(z)}{p^{2}-m_{n}^{2}} .
\end{aligned}
$$

The propagators are matrices in the flavor space and satisfy the coupled differential equations

$$
\begin{aligned}
i a^{-3} \delta(y-z) & =p^{2} P_{L L}-a\left(\hat{D}_{y}+M\right) P_{R L} \\
i a^{-3} \delta(y-z) & =p^{2} P_{R R}-a\left(-\hat{D}_{y}+M\right) P_{L R} \\
0 & =P_{L R}-a\left(\hat{D}_{y}+M\right) P_{R R} \\
0 & =P_{R L}-a\left(-\hat{D}_{y}+M\right) P_{L L},
\end{aligned}
$$

which can be derived using the equations of motions and the completeness relations for the profiles. To solve these equations we introduce auxiliary (hatted) propagators, separately for $y<z$ and for $y>z$

$$
\begin{aligned}
& P_{L L}^{<}(y, z)=a^{-2}(y) e^{M y} \Omega(y) \hat{P}_{L L}^{<}(y, z) \\
& P_{R R}^{<}(y, z)=a^{-2}(y) e^{-M y} \Omega(y) \hat{P}_{R R}^{<}(y, z) \\
& P_{L L}^{>}(y, z)=a^{-2}(y) e^{M y} \bar{\Omega}(y) \hat{P}_{L L}^{<}(y, z) \\
& P_{R R}^{>}(y, z)=a^{-2}(y) e^{-M y} \bar{\Omega}(y) \hat{P}_{R R}^{>}(y, z),
\end{aligned}
$$

where the Wilson rotation matrices act in the flavor space and are given by

$$
\Omega(y)=e^{i g_{5} \int_{0}^{y}\left\langle A_{5}\right\rangle} \quad \bar{\Omega}(y)=e^{i g_{5} \int_{L}^{y}\left\langle A_{5}\right\rangle} .
$$

The hatted propagators satisfy the second order differential equations

$$
\begin{aligned}
& {\left[a e^{-2 M y} \partial_{y}\left(a e^{2 M y} \partial_{y}\right)+p^{2}\right] \hat{P}_{L L}=0} \\
& {\left[a e^{2 M y} \partial_{y}\left(a e^{-2 M y} \partial_{y}\right)+p^{2}\right] \hat{P}_{R R}=0}
\end{aligned}
$$

that are valid for both $P^{<}$and $P^{>}$as long as $y \neq z$. The matching conditions at $y=z$ follow from Eq. (A2),

$$
\begin{aligned}
\Omega(L) \hat{P}_{L L}^{<}(z, z)= & \hat{P}_{L L}^{>}(z, z) \\
\left.\Omega(L) \partial_{y} \hat{P}_{L L}^{<}(y, z)\right|_{y=z}= & \left.\partial_{y} \hat{P}_{L L}^{>}(y, z)\right|_{y=z} \\
& -i a^{-3}(z) e^{-M z} \bar{\Omega}^{-1}(z) \\
\Omega(L) \hat{P}_{R R}^{<}(z, z)= & \hat{P}_{R R}^{>}(z, z) \\
\left.\Omega(L) \partial_{y} \hat{P}_{R R}^{<}(y, z)\right|_{y=z}= & \left.\partial_{y} \hat{P}_{R R}^{>}(y, z)\right|_{y=z} \\
& -i a^{-3}(z) e^{M z} \bar{\Omega}^{-1}(z) .
\end{aligned}
$$


The equations of motion (A6) and (A7) together with the matching conditions (A8) fully determine the chiralitydiagonal propagators, once the boundary conditions are specified. The chirality-flipping propagators can be calculated from the diagonal ones,

$$
\begin{gathered}
P_{L R}^{<}(y, z)=a^{-1}(y) e^{-M y} \Omega(y) \partial_{y} \hat{P}_{R R}^{<}(y, z) \\
P_{R L}^{<}(y, z)=-a^{-1}(y) e^{M y} \Omega(y) \partial_{y} \hat{P}_{L L}^{<}(y, z) \\
P_{L R}^{>}(y, z)=a^{-1}(y) e^{-M y} \bar{\Omega}(y) \partial_{y} \hat{P}_{R R}^{>}(y, z) \\
P_{R L}^{>}(y, z)=-a^{-1}(y) e^{M y} \bar{\Omega}(y) \partial_{y} \hat{P}_{L L}^{>}(y, z) .
\end{gathered}
$$

As an example, we compute the propagators in a simple toy model. Consider a 5D quark field $Q$ with a bulk mass $M$, transforming in the spinorial 4 representation of $S O(5)$ and having the $U(1)_{X}$ charge equal to $1 / 6$. The fermion contains fields with quantum numbers of the SM top and bottom quarks embedded as follows

$$
Q=\left(q, q^{c}\right)=\left(t, b, t^{c}, b^{c}\right) .
$$

The boundary conditions are

$$
q_{R}(0)=q_{L}^{c}(0)=0 \quad q_{R}(L)=q_{L}^{c}(L)=0 .
$$

This model is not realistic for several reasons, one being that it predicts degenerate top and bottom quarks, but it is simple enough to serve the illustration purpose.

First, following Ref. [17], we denote two independent solutions of Eq. (A6) as $C_{M}(y)$ and $S_{M}(y)$. We pick up these solutions such that they satisfy $C_{M}(0)=1, C_{M}^{\prime}(0)=0$, $S_{M}(0)=0, S_{M}^{\prime}(0)=p$. The notation is to stress the similarity to the familiar sines and cosines (to which these functions reduce for a flat warp factor and $M=0$ ). The warped generalization of $\sin ^{\prime}=\cos$ is $S_{M}^{\prime}(y)=$ $p a^{-1}(y) e^{-2 M y} C_{-M}(y), C_{M}^{\prime}(y)=-p a^{-1}(y) e^{-2 M y} S_{-M}(y)$. The generalization of $\sin ^{2}+\cos ^{2}=1$ is the Wronskian $S_{M}(y) S_{-M}(y)+C_{M}(y) C_{-M}(y)=1$. The explicit form of these solutions is of no relevance here; the only important property is that, at small momenta, we can approximate $C_{M}=1-\mathcal{O}\left(p^{2}\right), \quad S_{M}=p \int_{0}^{y} a^{-1}\left(y^{\prime}\right) e^{-2 M y^{\prime}}+\mathcal{O}\left(p^{3}\right)$. We also introduce the combinations $\bar{C}_{M}(y)=$ $a(L) e^{2 M L} p^{-1}\left[C_{M}(y) S_{M}^{\prime}(L)-S_{M}(y) C_{M}^{\prime}(L)\right], \quad \bar{S}_{M}(y)=$ $a(L) e^{2 M L}\left[-C_{M}(y) S_{M}(L)+S_{M}(y) C_{M}(L)\right]$, that satisfy simple boundary conditions on the IR brane: $\bar{C}_{M}(L)=1$, $\bar{C}_{M}^{\prime}(L)=0, \bar{S}_{M}(L)=0, \bar{S}_{M}^{\prime}(L)=p$.

Armed with this formalism, we write the hatted propagators as

$$
\begin{array}{ll}
\hat{P}_{L L}^{<q a}=C_{M}(y) c_{L}^{<q a}(z) & \hat{P}_{L L}^{>q a}=\bar{C}_{M}(y) c_{L}^{>q a}(z) \\
\hat{P}_{L L}^{<q^{c} a}=S_{M}(y) c_{L}^{<q^{c} a}(z) & \hat{P}_{L L}^{>q^{c} a}=\bar{S}_{M}(y) c_{L}^{>q^{c} a}(z),
\end{array}
$$

where $a=q, q^{c}$. This form is dictated by the boundary conditions (A12). Now we plug this into the matching Eq. (A8) and solve for the coefficient $c^{a b}(z)$. In particular, we find

$$
c_{L}^{q^{c} q}(0)=\frac{\sin (\tilde{v} / f)}{2 p} \frac{1-2 S_{M}(L) S_{-M}(L)}{S_{M}(L) S_{-M}(L)-\sin ^{2}(\tilde{v} / 2 f)} .
$$

For our purpose, we need only these coefficients since $\operatorname{Tr} T^{\hat{a}} P_{R L}(0,0)=P_{R L}^{q^{c} q}(0,0) / \sqrt{2}$ and, from Eq. (A9), $P_{R L}^{q^{c} q}(0,0)=-p c_{L}^{q^{c} q}(0)$. Poles of the propagator occur at $S_{M}(L) S_{-M}(L)-\sin ^{2}(\tilde{v} / 2 f)$, which determines the fermionic resonance spectrum. The limit $p^{2} \rightarrow 0$ is achieved by setting $S_{ \pm M} \rightarrow 0$. We find

$$
\operatorname{Tr}\left[\sqrt{2} T^{\hat{a}} P_{R L}(0,0)\right]=2 \frac{\cos (\tilde{v} / 2 f)}{2 \sin (\tilde{v} / 2 f)} .
$$

We have exposed the factor of 2 to stress that the formula includes contributions of degenerate top and bottom KK towers. Thus, in this toy-model, the contribution of the top quark tower is equal to $R^{1 / 2}=\cos ^{2}(\tilde{v} / 2 f)$ that of the SM top quark.

In the realistic models, the propagator is determined according to the same algorithm; the computation is just a tad more tedious.

\section{APPENDIX B: SO(5) GENERATORS}

$S O(5)$ has 10 generators: $T_{L}^{a}$ form the $S U(2)_{L}$ subgroup, $T_{R}^{a}$ form the $S U(2)_{R}$ subgroup, and the remaining four generators are denoted by $T_{C}^{b}$. The commutation relations,

$$
\begin{gathered}
{\left[T_{L}^{a}, T_{L}^{b}\right]=i \epsilon^{a b c} T_{L}^{c} \quad\left[T_{R}^{a}, T_{R}^{b}\right]=i \epsilon^{a b c} T_{R}^{c}} \\
{\left[T_{L}^{a}, T_{R}^{b}\right]=0} \\
{\left[T_{C}^{a}, T_{C}^{b}\right]=\frac{i}{2} \epsilon^{a b c}\left(T_{L}^{c}+T_{R}^{c}\right) \quad\left[T_{C}^{a}, T_{C}^{4}\right]=\frac{i}{2}\left(T_{L}^{a}-T_{R}^{a}\right)} \\
{\left[T_{L, R}^{a}, T_{C}^{b}\right]=\frac{i}{2}\left(\epsilon^{a b c} T_{C}^{c} \pm \delta^{a b} T_{C}^{4}\right)} \\
{\left[T_{L, R}^{a}, T_{C}^{4}\right]=\mp \frac{i}{2} T_{C}^{a} .}
\end{gathered}
$$

The smallest nontrivial $S O(5)$ representation is the spinorial one denoted as 4 . The five $4 \times 4$ gamma matrices of $S O(5)$,

$$
\begin{gathered}
\Gamma^{a}=\left[\begin{array}{cc}
0 & \sigma^{a} \\
\sigma^{a} & 0
\end{array}\right] \quad \Gamma^{4}=\left[\begin{array}{cc}
0 & -i \\
i & 0
\end{array}\right] \\
\Gamma^{a}=\left[\begin{array}{cc}
1 & 0 \\
0 & -1
\end{array}\right] .
\end{gathered}
$$

The generators,

$$
\begin{array}{cc}
T_{L}^{a}=\frac{1}{2}\left[\begin{array}{cc}
\sigma^{a} & 0 \\
0 & 0
\end{array}\right] & T_{R}^{a}=\frac{1}{2}\left[\begin{array}{cc}
0 & 0 \\
0 & \sigma^{a}
\end{array}\right] \\
T_{C}^{a}=\frac{i}{2 \sqrt{2}}\left[\begin{array}{cc}
0 & \sigma^{a} \\
-\sigma^{a} & 0
\end{array}\right] & T_{C}^{4}=\frac{1}{2 \sqrt{2}}\left[\begin{array}{ll}
0 & 1 \\
1 & 0
\end{array}\right] .
\end{array}
$$

They are normalized as $\operatorname{Tr} T^{\alpha} T^{\beta}=(1 / 2) \delta^{\alpha \beta}$. The $T^{3}$, s of 
$S U(2)_{L} \times S U(2)_{R}$ are diagonal in this basis. Thus, we can easily see how $S U(2)_{L} \times S U(2)_{R}$ quantum numbers are embedded in 4,

$$
q=\left[\begin{array}{l}
q_{+0} \\
q_{-0} \\
q_{0+} \\
q_{0-}
\end{array}\right] \quad \mathbf{4}=(\mathbf{2}, \mathbf{1}) \oplus(\mathbf{1}, \mathbf{2}) .
$$

The Wilson rotation matrix,

$$
\exp \left(i x T_{C}^{4}\right)=\left[\begin{array}{cc}
\cos (x / 2 \sqrt{2}) & i \sin (x / 2 \sqrt{2}) \\
i \sin (x / 2 \sqrt{2}) & \cos (x / 2 \sqrt{2})
\end{array}\right] .
$$

The fundamental $S O(5)$ representation is denoted as 5 . The generators can be chosen as,

$$
\begin{array}{ll}
T_{L, i j}^{a}=-\frac{i}{2}\left[\frac{1}{2} \epsilon^{a b c}\left(\delta_{i}^{b} \delta_{j}^{c}-\delta_{j}^{b} \delta_{i}^{c}\right)+\left(\delta_{i}^{a} \delta_{j}^{4}-\delta_{j}^{a} \delta_{i}^{4}\right)\right] & a=1 \ldots 3 \\
T_{R, i j}^{a}=-\frac{i}{2}\left[\frac{1}{2} \epsilon^{a b c}\left(\delta_{i}^{b} \delta_{j}^{c}-\delta_{j}^{b} \delta_{i}^{c}\right)-\left(\delta_{i}^{a} \delta_{j}^{4}-\delta_{j}^{a} \delta_{i}^{4}\right)\right] & a=1 \ldots 3 \\
T_{C, i j}^{\hat{a}}=-\frac{i}{\sqrt{2}}\left[\delta_{i}^{\hat{a}} \delta_{j}^{5}-\delta_{j}^{\hat{a}} \delta_{i}^{5}\right] \quad \hat{a}=1 \ldots 4, &
\end{array}
$$

and are normalized as $\operatorname{Tr} T^{\alpha} T^{\beta}=\delta^{\alpha \beta}$.

The $T^{3}$ generators of the $S U(2)_{L} \times S U(2)_{R}$ subgroup are nondiagonal in this basis. The vector of $S O(5)$ can be expressed as a combination of eigenvectors of $T_{L}^{3} \times T_{R}^{3}$,

$$
Q=\frac{1}{\sqrt{2}}\left(\begin{array}{c}
q_{++}+q_{--} \\
i q_{++}-i q_{--} \\
q_{+-}+q_{-+} \\
i q_{+-}-i q_{-+} \\
\sqrt{2} q^{c}
\end{array}\right)
$$

where \pm denotes $\pm 1 / 2$. Thus, 5 contains a bifundamental and a singlet under $S U(2)_{L} \times S U(2)_{R}$.

The Wilson rotation matrix,

$$
e^{i x T_{C}^{4}}=\left[\begin{array}{ccccc}
1 & 0 & 0 & 0 & 0 \\
0 & 1 & 0 & 0 & 0 \\
0 & 0 & 1 & 0 & 0 \\
0 & 0 & 0 & \cos (x / \sqrt{2}) & \sin (x / \sqrt{2}) \\
0 & 0 & 0 & -\sin (x / \sqrt{2}) & \cos (x / \sqrt{2})
\end{array}\right] .
$$

We also show how these matrices operate in the subspace $\left(q_{+-}, q_{-+}, q^{c}\right)$,

$$
\begin{aligned}
T_{C}^{4} & =\frac{1}{2}\left[\begin{array}{ccc}
0 & 0 & -1 \\
0 & 0 & 1 \\
-1 & 1 & 0
\end{array}\right] \\
e^{i h T_{C}^{4}}= & {\left[\begin{array}{ccc}
\frac{1+\cos (h / \sqrt{2})}{2} & \frac{1-\cos (h / \sqrt{2})}{2} & -i \frac{\sin (h / \sqrt{2})}{\sqrt{2}} \\
\frac{1-\cos (h / \sqrt{2})}{2} & \frac{1+\cos (h / \sqrt{2})}{2} & i \frac{\sin (h / \sqrt{2})}{\sqrt{2}} \\
-i \frac{\sin (h / \sqrt{2})}{\sqrt{2}} & i \frac{\sin (h / \sqrt{2})}{\sqrt{2}} & \cos (h / \sqrt{2})
\end{array}\right] . }
\end{aligned}
$$

[1] H. M. Georgi, S. L. Glashow, M. E. Machacek, and D. V. Nanopoulos, Phys. Rev. Lett. 40, 692 (1978); S. Dawson, Nucl. Phys. B359, 283 (1991); M. Spira, A. Djouadi, D. Graudenz, and P. M. Zerwas, Nucl. Phys. B453, 17 (1995).

[2] R. V. Harlander and W. B. Kilgore, Phys. Rev. Lett. 88, 201801 (2002); C. Anastasiou and K. Melnikov, Nucl. Phys. B646, 220 (2002).

[3] N. S. Manton, Nucl. Phys. B158, 141 (1979); Y. Hosotani, Phys. Lett. 126B, 309 (1983); Ann. Phys. (Paris) 190, 233 (1989); H. Hatanaka, T. Inami, and C. S. Lim, Mod. Phys. Lett. A 13, 2601 (1998).

[4] R. Contino, Y. Nomura, and A. Pomarol, Nucl. Phys. B671, 148 (2003).
[5] K. Agashe, R. Contino, and A. Pomarol, Nucl. Phys. B719, 165 (2005).

[6] Y. Hosotani, S. Noda, Y. Sakamura, and S. Shimasaki, Phys. Rev. D 73, 096006 (2006).

[7] Y. Sakamura and Y. Hosotani, Phys. Lett. B 645, 442 (2007).

[8] M. S. Carena, E. Ponton, J. Santiago, and C. E. M. Wagner, Phys. Rev. D 76, 035006 (2007).

[9] G. F. Giudice, C. Grojean, A. Pomarol, and R. Rattazzi, J. High Energy Phys. 06 (2007) 045.

[10] Y. Hosotani and Y. Sakamura, Prog. Theor. Phys. 118, 935 (2007).

[11] A. Falkowski, S. Pokorski, and J.P. Roberts, J. High 
Energy Phys. 12 (2007) 063.

[12] R. Contino, L. Da Rold, and A. Pomarol, Phys. Rev. D 75, 055014 (2007).

[13] I. Low, http://www.lns.cornell.edu/ eotcor07/budapest_ ianlow.pdf; I. Low and R. Rattazzi (unpublished).

[14] R. Contino and A. Pomarol, J. High Energy Phys. 11 (2004) 058.

[15] K. Agashe, A. Delgado, M. J. May, and R. Sundrum, J. High Energy Phys. 08 (2003) 050; A. Delgado and A. Falkowski, arXiv:hep-ph/0702234.

[16] K. Agashe, R. Contino, L. Da Rold, and A. Pomarol, Phys. Lett. B 641, 62 (2006).

[17] A. Falkowski, Phys. Rev. D 75, 025017 (2007).

[18] R. Barbieri, B. Bellazzini, V.S. Rychkov, and A. Varagnolo, Phys. Rev. D 76, 115008 (2007).

[19] A.D. Medina, N.R. Shah, and C.E.M. Wagner,
arXiv:0706.1281.

[20] A. Falkowski, arXiv:0710.4050.

[21] E. Katz, A. E. Nelson, and D. G. E. Walker, J. High Energy Phys. 08 (2005) 074.

[22] R. Contino, T. Kramer, M. Son, and R. Sundrum, J. High Energy Phys. 05 (2007) 074.

[23] J. R. Ellis, M. K. Gaillard, and D. V. Nanopoulos, Nucl. Phys. B106, 292 (1976).

[24] T. Han, H. E. Logan, B. McElrath, and L. T. Wang, Phys. Lett. B 563, 191 (2003); 603, 257(E) (2004); C. R. Chen, K. Tobe, and C. P. Yuan, Phys. Lett. B 640, 263 (2006).

[25] F. J. Petriello, J. High Energy Phys. 05 (2002) 003.

[26] A. Djouadi and G. Moreau, Phys. Lett. B 660, 67 (2008).

[27] A. Djouadi, Phys. Lett. B 435, 101 (1998); R. Dermisek and I. Low, Phys. Rev. D 77, 035012 (2008). 